\title{
Mortality of a Pregnant Patient Diagnosed with COVID-19: A Case Report
}

\author{
Uday Mahajan ${ }^{1}$, Deepika Kapil ${ }^{2}$ \\ ${ }^{1}$ Department of Medicine, Dr Rajendra Prasad Govt. Medical College, Kangra at Tanda, \\ Himachal Pradesh, India \\ ${ }^{2}$ Medical Officer, Civil Hospital Nagrota Bagwan \\ Corresponding Author: Deepika Kapil
}

\begin{abstract}
On her presentation she was conscious pulse rate was 92 beats per minute and respiratory rate was 16/minute and decreased air entry bilateral lungs and coarse crepitations in both infrascapular and infraaxillary and mammary area- not maintaining saturation with spo2 69\% at room air. She was put on nonbreathable mask @ $15 \mathrm{~L} /$ minute and she achieved spo2of $92 \%$. Her fetal assessment was done using NST which was reactive. She was managed conservatively on antiviral, antibiotic, oxygen therapy by combination of high flow nasal cannula @60L/min at fio2 80\% along with NRM @15 $\mathrm{L} / \mathrm{min}$. She was planned for induction once stable and once weaned from high flow nasal cannula. However she kept deteriorating and became tachypneic, dehydrated and delirious. Her repeated investigation revealed rise in TLC of 11800/ul and falling albumin level of $2.6 \mathrm{~g} / \mathrm{dl}$ and rise in ferritin of $726 \mathrm{ng} / \mathrm{ml}$. Her subsequent ABG were suggestive of respiratory alkalosis.

Subsequently she was not maintaining saturation and she was intubated and she collapsed.
\end{abstract}

Keywords: Mortality, pregnant patient, COVID19 , respiratory alkalosis

\section{INTRODUCTION}

In December 2019, an outbreak caused by a new coronavirus was started in Wuhan, Hubei province of China that led to a pandemic emergence according to the World Health Organization (WHO) on March 11, 2020.[1] According to the phylogenetic studies, the pathogen was named severe acute respiratory syndrome coronavirus 2 (SARS-CoV-2) and the disease was called coronavirus disease 2019 (COVID-19). Reports have shown different signs among the patients with COVID-19 among which fever and cough were most common [2]. According to the data, several clinical outcomes such as sepsis, respiratory failure, acute respiratory distress syndrome (ARDS), septic shock, coagulopathy, acute cardiac injury, and acute kidney injury are significantly (all P-values $<0.0001$ ) higher in non-survivor patients compared to survivors. Thus, these outcomes have been suggested to be monitored more cautiously among the admitted patients [3]. So far, few reports have provided information on the clinical and imaging follow up of pregnant patients with COVID-19. Although, no mortality has been reported among their patients. [4-6] Herein, we report a pregnant patient diagnosed with COVID-19 who, unfortunately, did not survive. This report is going to address the data from admission until after the autopsy.

\section{CASE REPORT}

A 29 year female gravida three with one previous caesarean delivery for failed induction eight years back and one spontaneous abortion in previous pregnancy admitted at our institution at 37 weeks of pregnancy with chief complaints of shortness of breath for past three days and detected Covid positive via RTPCR testing. She was unvaccinated for her Covid status prior to admission. 
Historically she was a booked case at a private hospital. She presented with shortness of breath for three days not associated with fever or cough or rash. There was no nausea or vomiting/no history of headache/no history of swelling hands or feet. Her daily foetal movement count was adequate.

On her presentation she was conscious pulse rate was 92 beats per minute and respiratory rate was 16 /minute and decreased air entry bilateral lungs and coarse crepitations in both infrascapular and infraaxillary and mammary area- not maintaining saturation with spo2 $69 \%$ at room air. She was put on nonbreathable mask@15 L/minute and she achieved spo2of $92 \%$

She was managed with a clinical possibility of Covid related illness with bilateral pneumonitis with acute respiratory distress syndrome and started on injectable ceftriaxone/ tablet zinc/tablet calcium/ propped up position/ capsule doxycycline $100 \mathrm{mg}$ bd / injectable anticoagulantenoxaparin/injection dexamethasone $6 \mathrm{mg}$ i.v. once a day, injection remdesivir

Subsequently investigated heamoglobin $11.2 \mathrm{gm} / \mathrm{dl}$, tlc $10.10 \mathrm{ml} / \mathrm{ul} /$ platelet 1,92000/ with neutrophilia / INR .96 wnl/ liver function test revealed transamnitis SGOT 79> sgpt 43 with alkaline phosphatase $265 \mathrm{u} / \mathrm{l} /$ mean plasma glucose $108 \mathrm{mg} / \mathrm{dl} /$ renal function and electrolytes within normal limits.

Inflammatory markers $\mathrm{c}$ reactive protein $>=24<48$, serum ferritin $162.6 \mathrm{ng} /$ $\mathrm{ml}$ and procactonin $-.480<.5 \mathrm{ng} / \mathrm{ml}$ ruling out systemic infection.

On day two of her admission she became agitated and became tachypneic her saturation started deteriorating and documented 89\% at NRM @15 litre/minute. Her ABG revealed pH of 7.465 with pCo2 of $29.7 \mathrm{mmhg}$ with $\mathrm{PO} 2$ of $56 \mathrm{mmhg}$ indicating respiratory alkalosis.

She was shifted to high flow nasal cannula@60 litre /minute with an fio2 of $80 \%$. Her inflammatory markers were repeated with a LDH of $1160 \mathrm{U} / \mathrm{L}$, procalcitonin of .400 and D-Dimer was 0.5 ruling out sepsis and pulmonary embolism but deteriorating and deteriorating inflammatory markers.

Her fetal assessment was done using NST which was reactive. She was managed conservatively on antiviral, antibiotic ,oxygen therapy by combination of high flow nasal cannula @60L/min at fio2 80\% along with NRM @15 L/min .She was planned for induction once stable and once weaned from high flow nasal cannula. However she kept deteriorating and became tachypneic, dehydrated and delirious .Her repeated investigation revealed rise in TLC of $11800 / \mathrm{ul}$ and falling albumin level of 2.6 $\mathrm{g} / \mathrm{dl}$ and rise in ferritin of $726 \mathrm{ng} / \mathrm{ml}$. Her subsequent $\mathrm{ABG}$ were suggestive of respiratory alkalosis.

Subsequently she was not maintaining saturation and she was intubated and she collapsed.

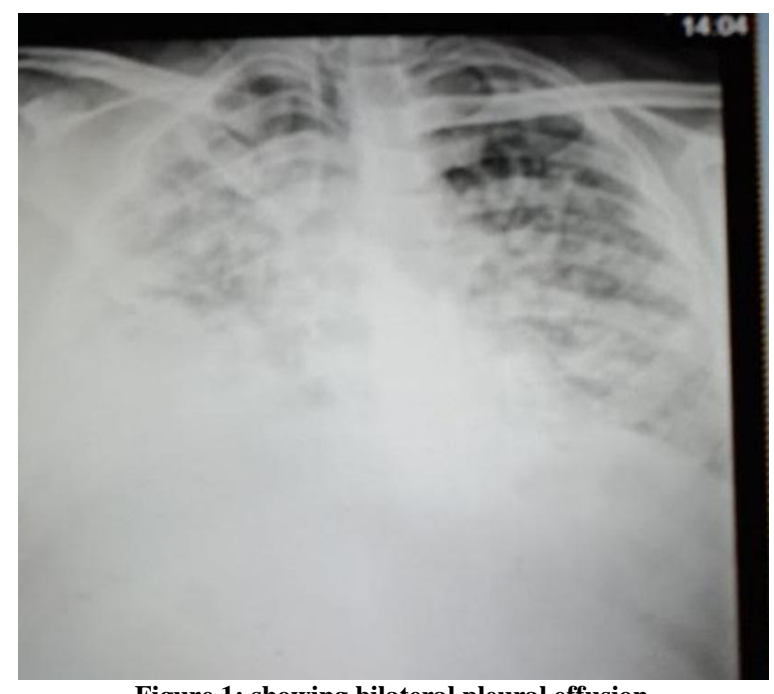

Figure 1: showing bilateral pleural effusion

\section{DISCUSSION}

Since 2000, two other outbreaks of coronaviruses have occurred other than COVID-19: Severe Acute Respiratory Syndrome (SARS) [10] and Middle East Respiratory Syndrome (MERS) [11]. Through the evaluations performed in the previous coronavirus outbreaks (SARS and MERS), pregnant women have been shown to be at increased risk of mortality, spontaneous miscarriage, preterm parturition, and intrauterine growth 
restriction. As it has been evaluated, the fatality rate of SARS and MERS among pregnant patients was $25 \%$ and $40 \%$, respectively $[10,11]$.

Another study evaluated 13 pregnant patients (two cases<and 11 cases $\geq 28$ week of gestation) diagnosed with COVID-19. They claimed the most common presentation among them to be fever $(77 \%)$ Of these 13 patients, $23 \%$ were discharged before labor and they continued their pregnancy normally. On the other hand, $77 \%$ undergone cesarean section due to several reasons including fetal distress $(3 / 10)$, premature rupture of the membrane or PROM (1/10), and stillbirth (1/10). Also, preterm labor was observed in $46 \%$ of all patients. One patient faced different complications such as multiple organ dysfunction. Due to the ARDS, she was intubated and put under mechanical ventilation in ICU. Also, she was diagnosed with acute kidney injury, acute hepatic failure, and septic shock. It is noteworthy to mention that this patient also had a stillbirth and according to the authors' statement, "Patient 6 was still in the support of Extracorporeal Membrane Oxygenation" (unavailable in our center) and no further information was given on the outcome of this patient [12]. Also, a study has evaluated the risk factors related to the mortality among survivor and non-survivor patients diagnosed with COVID-19. Authors have stated that sepsis, respiratory failure, ARDS, heart failure, septic shock, coagulopathy, acute cardiac injury, and acute kidney injury were significantly higher (p-values< 0.00001) in non-survivors compared to those discharged. Also, they have shown that the administration of corticosteroids in non-survivors was significantly higher than in survivor group. However, some other findings in their study such as non-invasive mechanical ventilation, high-flow nasal cannula oxygen therapy, and invasive mechanical ventilation were also significantly higher in non-survivors compared to the survivors ( $\mathrm{p}$-values< $0.00001)$. It is clear that taking these actions are all necessary in critically ill patients with COVID-19 and doesn't represent the worse outcome due to their action [3]. Taken together, there is not enough published evidence on COVID-19 (especially in pregnant patients) which help us to find the exact cause of death in this patient. Although, we have discussed the prominent findings of the patient (such as leukopenia, lymphopenia, elevated CRP, atypical imaging findings, and multi-organ failure) which have been known to associate with mortality in other published articles [3]

In our case there was sudden worsening of the patient condition and she was put on mechanical ventilation. The exact cause of death needs further investigation but due to worsening of respiratory findings we made the diagnosis of COVID related pneumonia.

\section{CONCLUSION}

In our case there was sudden deterioration of the patient which leads to cardiopulmonary arrest.

Conflict of Interest: All authors declare they have no conflict of interest.

\section{Financial Disclosure: Nil}

\section{Acknowledgement: None}

\section{Source of Funding: None}

\section{REFERENCES}

1. Organization WH. Rolling updates on coronavirus disease (COVID-19). https://wwwwhoint/emergencies/diseases/no vel-coronavirus-2019/events-as-theyhappen, Accessed date: 24 March 2020.

2. Guan W-j, Ni Z-y, Hu Y, et al. Clinical characteristics of coronavirus disease 2019 in China. N Engl J Med 2020. https://doi.org/10.1056/NEJMoa2002032. published online Feb 28.

3. Zhou F, Yu T, Du R, et al. Clinical course and risk factors for mortality of adult in patients with COVID-19 in Wuhan, China: a retrospective cohort study. Lancet 2020 . 
https://doi.org/10.1016/S0140-6736 (20)30566-3. published online March 11.

4. Wang1a X, Zhou2a Z, Zhang J, et al. A case of 2019 Novel Coronavirus in a pregnant woman with preterm delivery. Clin Infect Dis 2020. https://doi.org/10.1093/cid/ciaa 200. Published online Feb 28.

5. Schwartz DA. An analysis of 38 pregnant women with COVID-19, their newborn infants, and maternal-fetal transmission of SARS-CoV-2: maternal coronavirus infections and pregnancy outcomes. Arch Pathol Lab Med 2020. https://doi.org/ 10.5858/arpa.2020-0901-SA. Published online March 18.

6. Chen H, Guo J, Wang C, et al. Clinical characteristics and intrauterine vertical transmission potential of COVID-19 infection in nine pregnant women: a retrospective review of medical records. Lancet2020; 395:809-15.

7. Bai HX, Hsieh B, Xiong $Z$, et al. Performance of radiologists in differentiating COVID-19 from viral pneumonia on chest CT. Radiology 2020: 200823.

8. Kanne JP. Chest CT findings in 2019 novel coronavirus (2019-nCoV) infections from Wuhan, China: key points for the radiologist. Radiology 2020:200823.
9. Shi H, Han X, Jiang N, et al. Radiological findings from 81 patients with COVID-19 pneumonia in Wuhan, China: a descriptive study. Lancet Infect Dis 2020. https:// doi.org/10.1016/S1473-3099(20)30086-4. Published online Feb 24.

10. Wong SF, Chow KM, Leung TN, et al. Pregnancy and perinatal outcomes of women with severe acute respiratory syndrome. Am J Obstet Gynecol 2004; 191 : 292-7.

11. Assiri A, Abedi GR, Al Masri M, Bin Saeed A, Gerber SI, Watson JT. Middle East Respiratory Syndrome Coronavirus infection during pregnancy: a report of 5 cases from Saudi Arabia. Clin Infect Dis 2016; 63:951-3.

12. Liu Y, Chen H, Tang K, Guo Y. Clinical manifestations and outcome of SARS-CoV2 infection during pregnancy. J Infect 2020. https://doi.org/10.1016/j.jinf.2020.02.028. Published online March 4.

How to cite this article: Mahajan U, Kapil D. Mortality of a pregnant patient diagnosed with COVID-19: a case report. International Journal of Science \& Healthcare Research. 2021; 6(3): 66-69. DOI: https://doi.org/10.52403/ijshr. 20210712 